\title{
Quaderni
}

QUADERNI Communication, technologies, pouvoir

69 | Printemps 2009

Universités sous influence du numérique et du management

\section{Le dire managérial porté par les Tic : "démarche qualité » et « bonnes pratiques » dans l'enseignement supérieur}

\section{Christine Barats}

\section{(2) OpenEdition}

\section{Journals}

Édition électronique

URL : http://journals.openedition.org/quaderni/320

DOI : 10.4000/quaderni.320

ISSN : 2105-2956

Éditeur

Les éditions de la Maison des sciences de l'Homme

Édition imprimée

Date de publication : 1 mai 2009

Pagination : 75-91

\section{Référence électronique}

Christine Barats, «Le dire managérial porté par les Tic : " démarche qualité » et « bonnes pratiques » dans l'enseignement supérieur », Quaderni [En ligne], 69 | Printemps 2009, mis en ligne le 05 avril 2012, consulté le 30 avril 2019. URL : http://journals.openedition.org/quaderni/320 ; DOI : 10.4000/ quaderni.320 


\section{$D$ ossier}

\section{le dire managérial porté par les Tic : "démarche qualité" et "bonnes pratiques" dans l'enseignement supérieur}

\section{Christine Barats}

Maître de conférences Université Paris Descartes membre du Céditec
L'étude diachronique des textes ministériels sur l'intégration des Tic $^{1}$ (Technologies de l'information et de la communication) témoigne de la prise en compte, par l'autorité de tutelle, de préoccupations liées à la compétitivité attendue de l'enseignement supérieur français. Lors d'une précédente recherche portant sur un corpus de textes ministériels visant à l'intégration des Tic $^{2}$, nous avons ainsi observé des changements lexicaux et rhétoriques quant à la place des Tic pour l'enseignement. Il ne s'agit plus d'améliorer les conditions d'enseignement ou de proposer une formation tout au long de la vie grâce aux Tic, discours dominants jusqu'à la fin des années 90 ; les discours d'accompagnement mettent l'accent sur la concurrence et la compétitivité attendue du supérieur français ${ }^{3}$ grâce aux Tic ou par les Tic (les Tic incarnant le symbole de la compétitivité). La rhétorique de l'internationalisation éducative s'est imposée. Elle est caractérisée par une référence au « retard $»^{4}$, « retard » qui naturalise l'appel à la « compétitivité ». Citons à titre d'exemple, la lettre de mission de Valérie Pécresse (4/10/07), adressée à Henri Isaac, dans le cadre du rapport sur l'université numérique. Cet extrait illustre le primat accordé à la compétitivité du supérieur et au rôle des Tic. Il est représentatif de la rhétorique ministérielle sur la compétitivité et le retard : «Dans un contexte de mondialisation de l'enseignement supérieur, il apparaît que la France accuse un retard certain par rapport aux autres pays occidentaux pour l'accès à des cours en ligne et dans l'offre de formation à distance. Alors même que la maîtrise des technologies de l'information et de la communication apparaît de plus en plus comme un élément de compétitivité d'une Nation, ce retard dans la numérisation des formations supérieures risque 
d'obérer le développement de la France dans les années à venir $»^{5}$.

Ces notions (concurrence et compétitivité) sont explicitement ou implicitement évoquées dans les textes que nous avons étudiés visant à l'intégration des Tic, elles relèvent d'une approche en termes de rapports de force économiques dans un contexte international. Les réformes qui touchent au supérieur ( Lolf $^{6}$ et loi LRU ${ }^{7}$ ) ont amplifié ce phénomène en valorisant un mode de gouvernement des établissements basé sur la gestion, sur le management et la notion de performance. La « compétitivité » attendue grâce aux Tic s'inscrit donc dans un contexte global de réforme de l'État comme en témoigne la Lolf.

Or, si depuis la fin des années 60, l'injonction technologique était censée incarner la modernité, les discours d'accompagnement des Tic indiquent aujourd'hui la diffusion d'un impératif de « compétitivité » qui rencontre un autre discours dominant : celui de la " performance», de "l'économie de la connaissance $»^{8}$ et de «l'économie de l'immatériel»". Les discours sur « l'économie de l'immatériel » naturalisent le déterminisme et le progressisme technologiques tout en les combinant avec la financiarisation du monde ${ }^{10}$.

Dans ce contexte, nous étudierons les modalités discursives de diffusion et de circulation du dire managérial à partir de l'analyse de discours d'accompagnement visant à l'intégration des Tic. Notre principale hypothèse de travail porte sur la façon dont les outils Tic diffusent le dire managérial et contribuent à le banaliser et à le légitimer. Il est important de souligner que la dimension technique de l'informatique ou du management induit une certaine neutralité des outils techniques et «managériaux ». Isabelle Bruno s'est ainsi intéressée aux effets de codification et de prescription produits par le «benchmarking », objet technique dépolitisé en raison de la neutralité qui est, a priori, attachée à son usage. Elle a ainsi montré que la diffusion du "benchmarking » a constitué une technique de coordination des politiques nationales de recherche et d'éducation dans le cadre de la stratégie européenne de Lisbonne, tout en orientant l'action publique vers une finalité compétitive ${ }^{11}$.

Notre étude sur les modalités de diffusion du dire managérial a porté sur deux corpus liés aux Tic : - le site Educnet ${ }^{12}$, site officiel du ministère de l'Éducation Nationale et de l'Enseignement supérieur, "pour la généralisation de l'usage des TIC dans l'enseignement », afin d'étudier des discours à portée nationale et relayant la politique incitative de l'autorité de tutelle,

- l'intranet et les documents de la cellule Tice d'un établissement ${ }^{13}$ afin d'examiner des discours à un niveau local, celui d'une université.

Nous montrerons ainsi à partir de l'analyse de ces deux corpus (site Educnet et documents de la cellule Tice d'un établissement) que les Tic contribuent à diffuser le dire managérial, tout autant qu'elles contribuent à le produire. Les Tic sont en effet au centre d'une vision politique intégrant le management et la gestion pour l'enseignement supérieur, basée sur le modèle de l'entreprise commerciale. Après avoir rappelé notre conception de l'analyse de discours puis pris en compte les conditions de diffusion du dire managérial (légitimité de la gestion et du 
management), nous analyserons comment se diffuse le dire managérial à partir des résultats de l'analyse de nos deux corpus.

\section{Analyse du discours et hypothèses de recherche}

Notre conception de l'analyse du discours vise à articuler la matérialité discursive à ses conditions d'énonciation et de production. Pour le dire autrement, il s'agit d'articuler l'analyse du discours à une démarche sociologique et ethnométhodologique, comme l'envisage Alice Krieg-Planque avec la notion d' "observable en discours » qui sous-tend l'idée «que les discours peuvent être un lieu d'observation des pratiques qui ont contribué à les produire $»^{14}$. Il ne s'agit ni de réduire le discours à une matérialité discursive, reflet d'un contenu à saisir, ni de l'hypertrophier en le réifiant et en négligeant ses conditions d'énonciation, de production et de diffusion ; ni encore d'évacuer le sujet, l'acteur ou l'institution comme instances de prise en charge et de légitimation des discours. Il s'agit au contraire de dépasser les deux écueils de « « l'épaisseur» ou de « l'opacité » des phénomènes discursifs », comme le soulignent Claire Oger et Caroline Ollivier-Yaniv ${ }^{15}$, en articulant les « énoncés produits » aux conditions endogènes et exogènes de leur production, c'est-à-dire en tant qu'énoncés socialement, institutionnellement et historiquement situés. Pour ne prendre qu'un exemple, citons le ministère de l'Enseignement supérieur qui représente pour les établissements une autorité dont les représentants ministériels tirent leur légitimité pour organiser et dire la manière d'intégrer les Tic dans le cadre de la politique contractuelle. C'est donc la politique du ministère telle qu'elle se donne à lire et s'affiche dans son organisation et dans ses textes que nous avons analysée lors d'une précédente recherche ${ }^{16}$. Précisons qu'au sein même de l'entité «ministère », les acteurs qui participent au travail d'écriture de ces textes peuvent avoir des visions parfois différentes ${ }^{17}$. Les positions défendues dans les textes peuvent donc ne pas être intégralement partagées par les acteurs ou les experts. Cependant, d'un point de vue diachronique, les textes ministériels destinés aux établissements dans le cadre de la procédure quadriennale constituent une mise en mots officielle et incitative de la façon d'envisager les Tic dans l'enseignement supérieur français. Notons que ces incitations constituent des opportunités objectives de financement pour les établissements. Le recours aux entretiens, c'est-à-dire aux sources provoquées, s'avère donc indispensable pour 《 renseigner les textes $»^{18}$ et tenir compte de leurs conditions de production. Il peut s'agir également de compléter l'analyse du discours en mettant au jour des profils d'acteurs à partir d'un travail sociographique. Notre conception de l'analyse du discours vise à éviter la tentation anthropomorphique ou exégétique, en tenant compte des lieux de discours, de leur dimension contraignante et des rapports de force y afférents afin de constituer des corpus d'étude pluriels (textes, entretiens, sociographie des acteurs), liés à nos hypothèses de travail.

\section{Légitimité de la gestion et du management et diffusion du dire managérial}

Les contraintes de cet article ne nous permettent pas d'approfondir et de discuter la datation quant à la prise en compte des impératifs de gestion et à la diffusion des techniques gestionnaires (i.e. les indicateurs et les notions). Cependant, 
une historicisation des discours et notions de la gestion voire des pratiques gestionnaires montre que leur diffusion en France est liée au plan Marshall et à l'après-guerre (L. Boltanski, 1981). Des consultants et des experts américains en gestion et en management vont former les cadres de l'industrie en contrepartie de l'intervention financière américaine. En France, si les questions d'organisation dans l'entreprise n'étaient pas forcément désignées par les termes de " gestion » et de « management», leur pic de visibilité se situe dans les années 90 pour le secteur privé. La présence de la gestion et du management s'observe dans l'administration par des changements de vocabulaire et de techniques, ces dernières sont en effet censées garantir la transparence. Il s'agirait de passer d'une « culture de moyens » à une « culture de résultats ». « La fixation d'objectifs précis et d'indicateurs mesurables doit garantir scientifiquement l'avènement de l'efficiency " (Forest : 16). Un autre élément quant à la légitimité accordée aux discours et aux techniques gestionnaires en France peut s'observer par le prisme des disciplines nationalement reconnues par le conseil national des universités (CNU). La gestion constituera la $6^{\text {ème }}$ section du CNU, se dissociant de l'économie ${ }^{19}$ (début des années 70) et intégrant le management. Parallèlement, les formations en gestion vont se multiplier et l'offre de formation se développer, confirmant la légitimité accordée aux techniques de gestion et de management. «L'encyclopédie de gestion $»^{20}$ de 1989 indique dans un article de Christian Vulliez que le «système éducatif est probablement l'un des derniers secteurs où les préoccupations " gestionnaires » aient pénétré».

Priorités ministérielles pour l'enseignement supérieur depuis 1997, les Tic constituent un levier pour la mise en œuvre d'un pilotage du supérieur français tel que l'induisent la Lolf et la loi dite LRU. En effet, les Tic comme outil de pilotage portent et sont portées par les réformes qui touchent au supérieur et qui supposent des outils techniques afin de mettre en œuvre le système de contrôle de gestion prôné pour le pilotage des universités. Ces changements liés au financement des universités et aux relations entre l'État et les universités s'accompagnent de changements notionnels et organisationnels. Citons pour exemple la généralisation de la notion de « système d'information » qui remplace la notion de « système ou service informatique ». Yves Chevalier montre ainsi que l'étude généalogique de la notion de « système d'information » renvoie aux questions de pouvoir et de pilotage, il réfute une approche «naïve » et réductrice qui appréhenderait les « systèmes d'information » comme objet technico-technique, occultant la dimension organisationnelle et politique qui sous-tend tout système technique d'information. Les acteurs des « systèmes d'information » s'alimentent ainsi aux sources théoriques du New Public Management : «Bon nombre des notions que ces théories ont formalisées se retrouvent à l'œuvre dans l'appareil discursif et fonctionnel des systèmes d'information. Issues de modèles américains, les thèses majeures du New Public Management reposent sur l'idée que la gestion de l'administration peut être menée selon les mêmes critères et canons que ceux de l'entreprise privée $»^{21}$.

Nous avons ainsi observé l'émergence, que ce soit au niveau de l'autorité de tutelle ou d'un établissement, de notions jusqu'à présent absentes de la rhétorique ministérielle ou universitaire, 
comme les notions de « démarche qualité » ou de « bonnes pratiques ». Ces notions attestent de la diffusion et de la circulation du dire managérial. Il est important de rappeler que nous considérons que la diffusion de ces notions contribue à produire et à légitimer le dire managérial. C'est ce que nous proposons d'examiner à partir du site ministériel pour l'intégration des Tic : Educnet ainsi que les documents internes de la « cellule TICE » d'un établissement.

\section{De la compétitivité attendue dans le supérieur à la "démarche qualité » : des textes de ca- drage ministériels au site Educnet ou le dire managérial en ligne}

Depuis 1997, la promotion des Tic est une constante dans l'enseignement supérieur français. Les textes de cadrage de l'autorité de tutelle pour la politique contractuelle invitent ainsi les établissements à intégrer les Tic. Si les technologies et les outils Tic évoluent, ils actualisent une attente récurrente dans le champ des politiques publiques menées dans le domaine de l'enseignement, à savoir la promotion de techniques ou technologies dont les potentialités sont censées répondre aux préoccupations en matière d'éducation et d'enseignement. Depuis 1966 et le « plan calcul» de Pierre Messmer, puis le «Plan informatique pour Tous » de Laurent Fabius en 1985, les enjeux liés aux techniques ou technologies ont changé. S'il s'agissait de répondre à des enjeux industriels en terme d'équipement, les discours d'accompagnement mettaient l'accent sur la nécessité d'incarner le symbole de la «modernité $»^{22}$. Aujourd'hui, l'autorité de tutelle -à savoir le ministère de l'Enseignement supérieur- invite les acteurs du supérieur à une banalisation des outils Tic : la maitrise des compétences Tic est 《désormais indispensable $»^{23}$. La promotion et l'incitation ont cédé la place à une généralisation attendue. Cette généralisation est censée incarner la « compétitivité » d'une Nation.

Dans le cas des textes de cadrage de la politique contractuelle consacrés aux Tic, c'est « le contexte de plus en plus pressant » qui justifie l'introduction des Tic. Autrement dit, il s'agit ici d'une référence implicite à la compétitivité attendue du supérieur. Cette référence à la « compétitivité » va s'imposer dans les textes ministériels ${ }^{24}$. Elle caractérise aujourd'hui la rhétorique ministérielle sur la promotion et l'appropriation des $\mathrm{Tic}^{25}$.

Les différents rapports officiels ou les lettres de mission mettent majoritairement l'accent sur le « retard $»^{26}$ technologique ou la « menace » des conséquences de ce retard, tandis que les textes de cadrage liés à la politique contractuelle mettent en avant les « avantages » ou « les promesses » liés aux Tic.

L'outil technique est censé incarner la « compétitivité » et l'étude des discours montre une naturalisation du progrès technologique, progrès présenté comme inévitable d'où l'acceptation de la compétition comme contexte national et international.

La multiplication des modalités de diffusion des textes (courriers, notes, rapports, sites, blogs...) a contribué à renforcer la notoriété du site Educnet, site officiel du ministère de l'Éducation Nationale et de l'Enseignement supérieur, « pour la généralisation de l'usage des TIC dans l'enseignement $»$. Ce site est actuellement le $11^{\mathrm{e}}$ 
site gouvernemental en terme de fréquentation ${ }^{27}$. Sans présumer d'une réelle notoriété au sein de l'ensemble de la communauté du supérieur, ce site constitue un corpus de textes sur le thème des Tic destinés aux établissements. Les destinataires sont principalement les acteurs « TIC » et «TICE » (« TIC pour 1'Enseignement» ou « dans l'Enseignement ») du supérieur (chargés de missions, personnels enseignants et nonenseignants). Deux rubriques de la page d'accueil situées dans le bandeau horizontal ainsi que dans le bandeau vertical s'intitulent «Pour les enseignants » et indiquent l'identité du principal co-énonciateur escompté : les enseignants. Le nom du «ministère de l'Éducation Nationale, ministère de l'Enseignement supérieur et de la Recherche », situé en haut à gauche sous « Educnet », constitue une signature institutionnelle et officielle de ce site qui contribue à écrire et mettre en mots la politique publique dans le domaine des Tic.

Contrairement aux textes de cadrage du ministère que nous avons étudiés et qui sont marqués par un style et un lexique incitatifs (« inviter», « encourager », « contribuer $»)^{28}$ car ils rendent compte du mode de négociation et donc de communication entre le signataire des textes et les destinataires (président d'établissement et son équipe), les textes disponibles sur le site Educnet sont moins incitatifs et plus informatifs (destinataires potentiels : ensemble des personnels). La fonction que nous pouvons attribuer à ce site est une fonction d'administration de la preuve de la généralisation attendue de l'usage des Tic. En effet, cette administration de la preuve se fait par la publicisation de réalisations ou de manifestations (colloques, journées d'échanges...) liées aux Tic. Dans le prolongement de notre étude diachronique des textes de cadrage ministériels, ce site constitue donc une autre mise en mots de la politique d'incitation pour l'intégration des Tic, une incitation par l'exemple, les exemples valant pour preuve de la généralisation des Tic. Nous considérons qu'il s'agit également de textes d'accompagnement, dans la mesure où l'instance de prise en charge des discours demeure l'autorité de tutelle. Cependant, si les textes de cadrage s'avèrent prospectifs dans la mesure où ils sont censés dire et impulser la politique ministérielle dans ce domaine, les textes disponibles sur le site d'Educnet sont soit rétrospectifs (compte rendu d'événements ayant eu lieu) soit informatifs (événements à venir). La place, sur la page d'accueil du site, accordée à la notion de « Une ${ }^{29}$ emprunte à la logique d'un journal d'informations (informations rétrospectives et annonces d'événements à venir). Les informations figurant en « Une » mettent ainsi l'accent sur les projets, événements liés aux projets Tic. Il est intéressant de noter qu'à une même date (janvier 2009) les sites institutionnels liés au champ de l'enseignement et que nous appréhendons en tant que « vitrines » empruntent tous le terme et la notion de «Une» (cf. Site de Valérie Pécresse (http://www.enseignementsup-recherche.gouv. $\mathrm{fr} /$ ), le site consacré aux réformes du supérieur et à la « nouvelle université » : http://www.nouvelleuniversite.gouv.fr/, et le site du ministère de l'Éducation nationale (http://www.education. gouv.fr/).

Le style impersonnel domine au niveau de la page d'accueil du site Educnet. Les verbes d'action qui sont présents sur le site sont majoritairement à l'infinitif (« s'abonner», « télécharger »). Il y 
a une absence d'adresse directe aux co-énonciateurs.

La rubrique « Pour les enseignants » propose un espace consacré au «supérieur». Dans cet espace, une rubrique « des outils pour vos projets » indiquent la présence d'une interpellation directe du co-énonciateur ( « vos projets »). Le site a donc une dimension informationnelle et également organisationnelle, voire méthodologique, car il propose un certain nombre d'outils pour les porteurs de projet Tic. Les outils proposés dans les rubriques « actions transversales » et « des outils pour vos projets » indiquent des emprunts lexicaux à la gestion et au management ( « gestion de projet », « référentiel », « bonne pratique »...). Ces emprunts langagiers induisent des changements quant aux méthodes de travail et au rapport des enseignants aux formations dispensées. La notion de « maquette de diplôme » cède la place à la notion de « gestion de projet » qui induit l'animation d'équipe et implique l'appropriation de la notion de « bonne pratique ». Ainsi, les formules impersonnelles cèdent la place aux formules personnalisantes visant à associer les lecteurs, acteurs supposés de la politique ministérielle. Depuis le début de l'année 2006, de nombreux outils issus de la gestion et du management sont ainsi disponibles : « kit de gestion de projet $»^{30}$, « guide de la qualité » ${ }^{31}$, outils et notions fréquents dans le champ entrepreneurial.

Exemple du kit de gestion de projet :

« La Direction de la technologie-SDTICE a mis en place, en juin 2004, une cellule d'appui aux projets «Université numérique en région 》 (UNR) qui a pour mission de faciliter et accompagner la mise en auvre des actions prévues dans les contrats d'objectifs. Après avoir recensé les attentes des chefs de projets UNR, cette cellule d'appui a jugé opportun de répondre en priorité à deux besoins essentiels :

- la mise en réseau des UNR et la mutualisation des expériences, par la création d'un site collaboratif et l'organisation de journées thématiques ; - un apport en méthodologie pratique en matière de gestion de projet. Le kit " gestion de projet UNR » repose sur un référentiel, ensemble cohérent et complet de principes et de bonnes pratiques s'appliquant au contexte particulier des projets UNR dans vos universités. Cet ensemble comporte des outils opérationnels, adaptés de ceux en usage dans des projets réels (dont certains d'envergure), et a été testé et validé par un panel de chefs de projets avant mise à disposition $»$.

La référence au management est explicite dans le « guide de la qualité » proposé depuis février 2006, sur le site Educnet :

"Cette introduction a été élaborée pour aider les chefs de projet des Campus numériques à définir une démarche qualité adaptée aux besoins de leurs projets et de leur environnement. Elle propose une définition de ce que peut-être une démarche qualité, une méthode et un guide de questionnement $»$.

«Définition: À la différence d'une évaluation qui consisterait à produire un jugement sur une formation à partir de la comparaison entre le souhaité et l'observé, une démarche qualité vise une amélioration continuelle. C'est une composante du management d'un projet ou d'un système ».

Il en est de même dans le cas de Compétice (" gérer les compétences pour vos projets 
$T I C E »)$, autre outil en ligne disponible dans la rubrique « actions transversales ». Cet « outil d'accompagnement » propose une «boîte à outils » et des documents à télécharger. Y figurent des définitions du management de projet :

« Dans un projet TICE, qui est un projet de changement, le chef de projet doit faire le choix d'un mode de management, c'est-à-dire un mode de pilotage du projet et d'orchestration, en particulier des ressources humaines ».

Sont ensuite présentés quatre principaux modes de management d'Henri Fayol (1916) à Peter Senge (1990). Il est important de noter que ces outils ( "Compétice » et le " guide de la qualité ») sont présents sur le site Educnet depuis 2006 et qu'ils ont été regroupés pour être présentés sur le site comme les deux principaux « outils d'accompagnement » (site consulté en janvier 2009). La présence de ces outils dits d'accompagnement et la place qui leur est accordée confirment notre hypothèse, à savoir que les outils Tic contribuent à diffuser le dire managérial et ses notions.

\section{De l'administration de la preuve par l'exemple à l'exemplarité de la "démarche qualité " initiée par l'autorité de tutelle}

Il est important de souligner que l'organigramme de la SDTICE comporte depuis mai 2005 un responsable «qualité » (Jean Heutte ${ }^{32}$ ). Lors de la réorganisation de la sous-direction en septembre 2003, Benoît Sillard avait confié à Jean-François Pillou $^{33}$ un programme « qualité, veille et diffusion », ce qui confirme également la légitimité accordée à la gestion et au management dans le choix des profils des acteurs. Il était chargé de mener à bien une « démarche qualité » en vue d'une certification ISO 9001. Cette norme qui s'applique aux systèmes de gestion de la qualité, date de 2001. Elle est issue du secteur privé et constitue un indice supplémentaire de la diffusion des outils et notions du management privé dans le secteur public.

Une lettre du sous-directeur de la SDTICE, Jean-Yves Capul, datée du 17 décembre 2007, confirme «Jean Heutte dans ses fonctions de responsable qualité $»^{34}$. Si l'objet de ce courrier stipule : «Engagement pour le management par la qualité », il rappelle l'obtention en mai 2007 par la SDTICE de la « reconnaissance à valeur internationale par la certification de système de management qualité selon la norme ISO 9001, pour trois ans. » L'obtention de cette certification peut être appréhendée comme une mise en pratique du dire managérial. La publicisation sur le site Educnet de ce "processus qualité » mis en place par la SDTICE confirme la démarche incitative par l'exemple que nous avons observée sur le site. L'autorité de tutelle - par le biais de la certification ISO 9001 obtenue par la SDTICE - se positionne ainsi dans une posture d'exemplarité que la certification obtenue contribue à construire.

Que ce soit au niveau des outils du site Educnet ou du profil des acteurs de la SDTICE, nous pouvons noter une même légitimité accordée aux outils de gestion et de management. Ces notions attestent donc de la diffusion et de la circulation du dire managérial, tout autant qu'elles contribuent à produire et mettre en pratique le dire managérial dans le secteur public. Au niveau d'un établissement, une diffusion comparable du dire managérial et de ses outils est observable. 
Les «bonnes pratiques » dans un établissement ou les notions qui façonnent les institutions

À partir d'une étude monographique menée au sein d'un établissement du supérieur ${ }^{35}$, en particulier lors de la mise en place d'un espace de travail dit collaboratif ${ }^{36}$, nous nous sommes intéressés aux discours sur les Tic et aux logiques en présence. L'établissement étudié a mis en service entre 1999 et 2007 un portail intranet et un premier espace de travail dit collaboratif (ou environnement numérique de travail), destiné à environ 34900 personnes (dont 32000 étudiants, 1800 personnels enseignants et plus de 1100 personnels $\operatorname{IATOS}^{37}$ ). Depuis 2005, le ministère de tutelle préconise la mise en œuvre de solutions nationales, au détriment de solutions locales, pour les environnements numériques de travail (ENT), également parfois désignés par l'expression « bureau virtuel $»^{38}$. L'établissement étudié a annoncé en 2005 l'abandon de l'espace collaboratif mis en œuvre en 1999, au profit d'un environnement numérique de travail qui est l'une des quatre solutions nationales proposées et soutenues par le ministère. Nous avons mené une enquête par entretiens et par observations au sein de l'ensemble des composantes de l'établissement. Nous nous intéresserons aux textes mis en ligne par la cellule «TICE» de cet établissement.

\section{Caractéristiques de l'établissement étudié :}

- Effectif global :

34900 personnes, dont 32000 étudiants, 1800 personnels enseignants, 1100 personnels IATOS ;

- 10 UFR ou composantes réparties sur 12 sites distants.
Si au niveau national, c'est en 2001 que l'acronyme Tice (Technologies de l'information et de la communication pour l'enseignement ou éducative) s'est imposé, c'est en 2005 que l'établissement étudié l'a adopté avec la création d'une vice-présidence «TICE ». Parallèlement à cette reconnaissance au niveau du pilotage politique de cet établissement, une cellule «TICE » a été mise en place, indiquant une triple logique en œuvre : politique (engagement du président), organisationnelle (organigramme) et fonctionnelle (personnel ad hoc). Une même personne était responsable de ces deux fonctions, jusqu'en septembre 2008. Des tensions avec la Direction des Systèmes d'Information (DSI) ont cependant marqué cette nouvelle organisation.

La cellule « TICE » indique comme missions sur le portail de l'établissement dédié à la cellule :

«Les missions de la cellule TICE

Les missions de la cellule TICE de l'Université $X X X$ sont concentrées autour de trois axes:

- Le développement du travail coopératif dans l'enseignement :

- Favoriser la communication et les échanges d'informations.

- Mettre en valeur les initiatives.

- Dégager de bonnes pratiques.

- L’aide aux projets pédagogiques :

- Offrir une aide à la publication et à la diffusion électronique de documents et ressources pédagogiques.

- Animer des ateliers enseignants dont les programmes s'ajustent à leurs besoins pratiques.

- Aider «en situation» les enseignants, en formant aux outils leurs groupes d'étudiants.

- La modernisation de l'informatique pédagogique: Proposer des méthodes et outils 
multimédia, simples et plastiques adaptés à un cadre pédagogique :

- Développement de l'enseignement à distance (tutorat, accompagnement des étudiants),

- Méthodologie en matière de visioconférence et d'enregistrement de cours audiovisuels.

- Suivi du développement des politiques technologiques appliquées à l'enseignement supérieur $»^{39}$.

La notion de « bonne pratique » (« best practice ou best practices »), notion d'origine anglosaxonne, qui signifie une capitalisation par l'expérience est issue du «benchmark». Le « benchmark » est une analyse comparative issue du marketing et de la gestion de la qualité (management), il est parfois traduit par « intercomparaison » ou « parangonnage ». Il induit la notion de palmarès dans la mesure où il s'agit d'une mise en comparaison, parfois d'un étalonnage des performances. La notion de «bonne pratique » est une conséquence du «benchmarking » car elle induit une comparaison des pratiques afin de dégager la ou les bonnes pratiques. La notion de « benchmark » est directement issue du secteur professionnel privé. C'est l'entreprise Xerox qui l'a mise en place en 1980, dans le cas de la gestion des stocks. Le «benchmark» est actuellement un des principaux outils du dire managérial et il a fait l'objet d'une réappropriation dans le contexte de l'enseignement avec la mise en place de l'Espace Européen de la Recherche, comme nous l'avons souligné en introduction, il oriente cependant l'action politique vers une finalité compétitive ${ }^{40}$. Il est intéressant de noter que la notion de «bonne pratique » issue $d u$ « benchmark » est présente dans les projets proposés par la cellule «TICE » et véhiculée par les termes de " coopératif ", " échanges d'informations ». Si cet exemple souligne le poids des emprunts anglosaxons dans le domaine des Tic et de la gestion, il confirme les changements lexicaux et notionnels qui accompagnent le développement des Tic. Les « bonnes pratiques » sont présentées comme contribuant au travail de mutualisation et de «qualité » dans l'appropriation des Tic par le personnel enseignant de l'établissement et l'accessibilité du web. Comme nous l'avons observé précédemment, les notions de « qualité »et de « démarche qualité », issues du secteur privé et des normes ISO ont figuré en février 2006 sur le site du ministère, Educnet. Que ce soit au niveau du ministère ou de l'établissement étudié, les changements langagiers introduits par les Tic témoignent donc de la prégnance des discours issus de la gestion et du management dans le supérieur français, discours qui contribuent à façonner les institutions dans la mesure où ils importent des notions qui visent à une certaine organisation et conception du travail.

Parmi les ressources proposées sur le site de la cellule « TICE » de l'établissement étudié figure un « référentiel de bonne pratique », afin que l'enseignant adopte une posture réflexive pour valider la « qualité » de sa ressource pédagogique :

«Les éléments proposés ici serviront à la réalisation d'un référentiel de bonnes pratiques destiné aux enseignants. Cette grille de lecture méthodologique doit leur permettre de valider eux-mêmes la " qualité " de leurs ressources sur le plan de la forme. Cette liste est destinée à être classée en plusieurs niveaux ce qui explique que certaines informations paraissent redondantes ».

Comme nous le soulignions, l'outil Tic induit 
ainsi un nouveau lexique emprunté au champ de l'informatique et à celui du management ( « référentiel », « bonne pratique », « démarche qualité »), qui vise à modifier les pratiques, cependant, il s'avère difficile de présumer de l'appropriation de ces notions et de la nature des effets de réel qu'elles peuvent produire sur le long terme, nonobstant les changements organisationnels et fonctionnels en cours. Néanmoins, dans un contexte de réformes de l'État et du financement public, la croyance dans la légitimité et la scientificité de la gestion et du management contribue cependant à banaliser le dire managérial et ses outils, outils fondateurs des réformes de l'État et du mode de financement public des universités, basé sur « objectifs » et « résultats ». Porté par les Tic, le dire managérial induit un esprit gestionnaire et ses corollaires : compétitivité, performance, classements, indicateurs.

\section{Des discours... à l'esprit gestionnaire}

Les Tic sont ainsi au centre d'une vision politique intégrant le management et la gestion pour l'enseignement supérieur. Des résistances à ce discours dominant -dont la légitimité relève de la croyance accordée aux techniques de gestion et de management- sont observables dans certains discours syndicaux ou sur le site de l'association « Sauvons la recherche » qui reprennent pour les dénoncer les emprunts au champ de la gestion, de l'entreprise privée et du management. Au niveau de l'établissement étudié, nous avons observé des résistances qui contribuent à réaffirmer une identité propre au champ de l'enseignement et de la recherche. Plusieurs personnes nous ont ainsi indiqué que le changement d'ENT effectué en 2007 s'expliquait aussi par un frein idéologique ou culturel entre des outils commerciaux (le premier espace collaboratif était basé sur des technologies Microsoft) et des outils non commerciaux (liés aux logiciels dits libres). Une composante s'est ainsi opposée à l'espace collaboratif développé entre 2000 et 2003 car il reposait sur un environnement Microsoft (ASP-Active Serveur Page et serveur applicatif Microsoft.net). En France, dans l'enseignement, le positionnement par rapport à Microsoft s'avère être un élément structurant pour faciliter ou au contraire hypothéquer le développement d'outils Tic. Soufiane Rouissi (2005) qui a mené une enquête sur les pratiques déclarées face à l'environnement numérique de travail d'une communauté universitaire d'enseignants-chercheurs dans la région Aquitaine indique ainsi que les enseignants regrettent le choix des logiciels Microsoft, ils sont cependant majoritairement utilisés et «moins de $20 \%$ des enseignants-chercheurs ont cité spontanément un logiciel libre même si le nombre de logiciels cités témoigne d'une grande diversité des outils». Dans le cas de l'établissement étudié, le choix Microsoft a justifié le refus de la part d'une des composantes et de certains acteurs d'utiliser le premier espace collaboratif. Les outils proposés par la cellule Tice s'inscrivent dans une logique open source, conforme à ce positionnement propre au supérieur français. Ce clivage entre technologies marchandes ou non marchandes s'inscrit également dans l'imaginaire qui a porté Internet (Flichy, 2001) et la dimension utopique d'un réseau ouvert (Arpanet) : «Les pères fondateurs d'Internet n'imaginaient pas seulement un réseau de coopération et d'échange entre les machines (transfert de fichiers) et entre les hommes (messageries, newsgroup), mais aussi l'accès à un savoir universel » (Flichy, 2001, 75). 
Le world wide web (littéralement : la toile d'araignée mondiale) dans sa généalogie même renvoie à de nombreuses utopies et idéologies. Il semble que dans le cas du supérieur français, les positions vis-à-vis de Microsoft réactivent ces clivages et fonctionnent également comme une dimension identitaire propre au champ de l'enseignement et de la recherche. Les outils open source semblent davantage correspondre aux cadres d'interprétation du supérieur et à l'idéal qui le fonde d'accès démocratisé au savoir. Dans un contexte de réforme du supérieur et de l'État, le modèle de l'entreprise privée et ses outils discursifs et techniques semblent cependant se diffuser. Lors d'entretiens effectués avec des secrétaires généraux d'établissement à propos de la mise en place des systèmes d'information, j'ai pu mesurer la dimension sensible du dire managérial dans le supérieur. En effet, les hésitations et la prudence discursive, observables lors des entretiens, ont confirmé les enjeux politiques autour de ces questions présentées comme techniques. La technique - que ce soit les outils Tic ou les outils comptables - tend à naturaliser des visions du monde qu'elle porte ou actualise. Si au niveau des rapports officiels et des discours politiques, le management, l'évaluation, l'approche quantitative et comptable dominent, la situation diffère au sein des établissements. Ces discours dominants produisent cependant des effets de réel, que ce soit dans le crédit accordé à ces approches (croyance économique), ou à leurs outils (esprit gestionnaire). De plus, ils passent sous silence les débats sur le gain escompté d'heures en présentiel grâce aux Tic, et par suite d'heures de service d'enseignement. Les discours naturalisent la compétition et contribuent à occulter les débats sur les missions de la recherche ou de l'enseignement supérieur, la construction des critères ou indicateurs d'évaluation et la question de leur légitimité.

La question de la notoriété et de la visibilité des universités constitue aujourd'hui une préoccupation majeure des universités, comme en témoignent les changements de nom d'universités et les associations entre établissements ${ }^{41}$. Dans ce deuxième ordre de discours ${ }^{42}$ - où le faire, c'est dire $^{43}$-, les noms des établissements, entendus comme signature représentant une institution, font l'objet de changements afin de répondre à une logique de marque, indice supplémentaire des effets de réel des logiques managériales et entrepreneuriales dans le champ de l'enseignement et de la recherche. 


\section{$R \cdot \dot{E} \cdot F \cdot E \cdot R \cdot E \cdot N \cdot C \cdot E \cdot S$}

BARATS Christine, « Les mythes du supérieur à l'heure des TIC, analyse de la rhétorique ministérielle », Colloque EUTIC06, Université Libre de Bruxelles, septembre 2006.

BARATS Christine, « «Pour le prix d'un café par jour », analyse des logiques d'institutionnalisation des Tic par le ministère de l'Éducation Nationale dans l'enseignement supérieur français depuis 1988 », in Communication, $\mathrm{n}^{\circ} 25$ (2) Québec, Automne 2007.

BARATS Christine, « Rhétorique de l'internationalisation et effet des classements internationaux dans le supérieur français : " compétitivité » et notoriété des universités ", in actes du colloque JOCAIR, 27, 28 août 2008, Amiens, Hermès Lavoisier, pp. 365-379.

BRUNO Isabelle, A vos marques, prêts... cherchez !, la stratégie européenne de Lisbonne, vers un marché de la recherche, Éditions du croquant, savoir/agir, Paris, 2008.

BOLTANSKI Luc, "America, America », in ARSS, Volume 38, 1981, pp. 19-41.

BARON Georges-Louis et BRUILLARD Éric, L'informatique et ses usagers dans l'éducation, PUF, 1996.

BONNAFOUS Simone et TEMMAR Malika, L'Analyse de Discours en SHS, Ophrys, 2007. BOUCHARD Julie, «L'invention du retard comme rhétorique de l'insuffisance et du changement », in Mots, n 80, 2006.

CHEVALIER Yves, Système d'information et gouvernance, Éditions Modulaires Européennes, Belgique, 2008.

FLICHY Patrice, L'imaginaire d'Internet, La découverte, 2001.
FOREST Frédéric, « «L'entreprise État » et les discours sur la réforme des lois de finances », in Quaderni, ${ }^{\circ}$ 53, Hiver 2003-2004, pp.13-20. FOUCAULT Michel, L'ordre du discours, Gallimard, 1971.

KRIEG-PLANQUE Alice, « La notion d' « observable en discours ». Jusqu'où aller avec les sciences du langage dans l'étude des pratiques d'écriture journalistique ? », in Marcel Burger (dir.), L'analyse linguistique des discours médiatiques, Québec, Université de Laval, Éditions Nota Bene, 2008, pp. 59-81.

MIÈGE Bernard, La Société conquise par la communication. Tome III, Les Tic entre innovation technique et ancrage social, PUG, 2007.

MUSSELIN Christine, La longue marche des universités françaises, Paris, PUF, 2001.

MUSSO Pierre, « Une critique de l'« économie de l'immatériel» vue par le rapport JouyetLévy », in Quaderni, $\mathrm{n}^{\circ} 64$, automne 2007, pp. 81-88.

OGIEN Albert, L'esprit gestionnaire, Éditions de l'EHESS, 1995.

ROUISSI Soufiane, « Pratiques Déclarées D’une Communauté Universitaire D'enseignants-Chercheurs Face À L'environnement Numérique De Travail. » Colloque SIF, Paris, 12-13/12/2005. $<$ http://Sif2005.Mshparisnord.Org/Pdf/Rouissi. Pdf.

ZETLAOUI Tiphaine, « Société de la connaissance », in Quaderni, $\mathrm{n}^{\circ}$ 63, printemps 2007, « Nouveaux mots du pouvoir », pp. 26-27. 
1. Par commodité, nous utiliserons l'acronyme « Tic » en minuscule afin de nous démarquer des acronymes ou sigles que nous étudions, sans présumer pour autant une homogénéité de sens, Voir Miège 2007.

2. Étude des textes de cadrage du ministère de l'Enseignement supérieur depuis 1989, voir Christine Barats, «Rhétorique de l'internationalisation et effet des classements internationaux dans le supérieur français : " compétitivité » et notoriété des universités ", in actes du colloque JOCAIR, 27, 28 août 2008, Amiens.

3. Pour simplifier, nous emploierons «supérieur» pour « enseignement supérieur».

4. La référence au « retard » semble s'inscrire dans une rhétorique étudiée par Julie Bouchard marquée par un régime absolutiste " où le retard est établi comme un écart négatif entre la réalité et ce qui est tenu comme un idéal à atteindre dans l'échelle du progrès ». Voir Julie Bouchard, «L'invention du retard comme rhétorique de l'insuffisance et du changement », in Mots, n०80, 2006.

5. Rapport à Valérie Pécresse, L'université numérique, Henri Isaac, Mcf en sciences de gestion, Dauphine, décembre 2007. Souligné par nous.

6. Loi organique relative aux lois de finance, du 1er août 2001, application à l'administration au 1er janvier 2006. Ministère des Finances, «la Lolf en 4 clics», "un nouveau cadre budgétaire pour réformer l'État ", " elle met en place une gestion plus démocratique et plus performante », « un budget plus lisible pour tous », «34 missions, 133 programmes, 580 actions », réforme présentée comme technique et non pas politique, neutralisation de la dimension politique et naturalisation de la technique... Voir http://www.finances.gouv.fr/lolf/4clics/clic1.htm,
Voir Frédéric Forest, in Quaderni, n 53, hiver 2003 2004.

7. Loi relative aux Libertés et Responsabilités des Universités, du 10 août 2007, dite loi LRU.

8. Si l'expression "Société de la connaissance» est liée au sociologue Daniel Bell qui, dans les années 70 , prescrivait aux sociétés de fonder leur économie sur celle de la connaissance (Vers la société postindustrielle (1973), Paris, Laffont, 1976, pp. 5354), elle a été reprise dans les années 90 lors des réunions du G7 dans le contexte des « autoroutes de l'information ». L'expression s'est diffusée en France dans les années 2000 pour désigner le «projet idéel d'une société post-industrielle dans laquelle l'origine de la richesse et de la prospérité des Nations se situerait dans la production et la circulation des biens immatériels mis en trafic par les moyens des Tic», Tiphaine Zetlaoui, « Société de la connaissance », in Quaderni, $\mathrm{n}^{\circ}$ 63, printemps 2007, « Nouveaux mots du pouvoir », pp. 26-27.

9. Rapport de Jean-Pierre Jouyet et Maurice Lévy, «L'économie de l'immatériel, la croissance de demain », décembre 2006.

10. Voir l'analyse de P. Musso, «Une critique de l'«économie de l'immatériel» vue par le rapport Jouyet-Lévy », in Quaderni, n 64, automne 2007, pp. 81 et suivantes.

11. Isabelle Bruno, A vos marques, prêts... cherchez !, la stratégie européenne de Lisbonne, vers un marché de la recherche, Éditions du croquant, savoir/agir, Paris, 2008.

12. Ce site est actuellement le $11^{\mathrm{e}}$ site gouvernemental en terme de fréquentation, http://www.educnet. education.fr

13. Établissement pluridisciplinaire accueillant 32000 étudiants.

14. A. Krieg-Planque, "La notion d'« observable en discours ». Jusqu'où aller avec les sciences 
du langage dans l'étude des pratiques d'écriture journalistique? », in Marcel Burger (dir.), L'analyse linguistique des discours médiatiques, Éditions Nota Bene, 2008, pp. 59-81.

15. C. Oger, C. Ollivier-Yaniv, « Analyse du discours et sociologie compréhensive. Retour critique sur une pratique de recherche transdisciplinaire ", in S. Bonnafous et M. Temmar, L'Analyse de Discours en SHS, Ophrys, 2007, p. 49.

16. Sur l'approche ministérielle, voir C. Barats, « «Pour le prix d'un café par jour», analyse des logiques d'institutionnalisation des Tic par le ministère de l'Éducation Nationale dans l'enseignement supérieur français depuis 1989 », in Communication, 2007.

17. Ce que nous avons observé grâce à des entretiens effectués avec des acteurs du supérieur (chargés de mission ou responsables de structure).

18. Expression que nous empruntons à Alice KriegPlanque (op.cit., p. 60) et qui peut impliquer un recours aux entretiens pour renseigner les textes.

19. Qui s'était dissociée du droit plus tôt.

20. Christian Vulliez, « Gestion d'un établissement d'enseignement », Économisa, 1989, p. 1346.

21. Yves Chevalier, Système d'information et gouvernance, EME, Belgique, 2008, p. 69.

22. Baron, Bruillard, L'informatique et ses usagers dans l'éducation, PUF, 1996.

23. C2i, certificat informatique et internet. Cf. $\underline{\mathrm{BO}} \mathrm{du}$ $11 / 03 / 04$.

24. Voir Barats, « Rhétorique de l'internationalisation et effet des classements internationaux dans le supérieur français : « compétitivité » et notoriété des universités », op. cit.

25. L'acronyme TIC (technologies dites de l'information et de la communication) s'est imposé après le sigle NT (Nouvelles technologies) ou NTIC qui insistaient sur la dimension prétendument nouvelle de ces technologies. Dans le champ de l'enseignement supérieur, il cohabite avec l'acronyme TICE depuis le début des années 2000.

26. La référence au « retard» semble s'inscrire dans une rhétorique étudiée par Julie Bouchard marquée par un régime absolutiste "où le retard est établi comme un écart négatif entre la réalité et ce qui est tenu comme un idéal à atteindre dans l'échelle $d u$ progrès». Voir Julie Bouchard, «L'invention du retard comme rhétorique de l'insuffisance et du changement », in Mots, $\mathrm{n}^{\circ}$ 80, 2006.

27. Source site Educnet, www.educnet.education.fr (janvier 2008) avec 592229 visiteurs mensuels en novembre 2007 et des pics à plus de 680000 visiteurs mensuels (janvier et mars 2008), http://www2. educnet.education.fr/sections/infosite/editeur-site.

28. Voir Barats, «Les mythes du supérieur à l'heure des TIC, analyse de la rhétorique ministérielle », in Colloque EUTIC06, Université Libre de Bruxelles, septembre 2006.

29. D'autres sites ministériels, consultés en janvier 2009, (économie, finances, budget...) utilisaient le terme « actualités ».

30. Présenté dans la rubrique «des outils pour vos projets », consultée en janvier 2009.

31. Présenté dans la rubrique « actions transversales », consultée en janvier 2009.

32. Titulaire d'un DESS en sciences de l'information et de la communication, "Communautés virtuelles et management de l'intelligence collective via les réseaux numériques », université de Limoges.

33. Ingénieur de formation, il a créé en février 1999, le site « Commentçamarche », dont l'actuel PDG est Benoît Sillard.

34. Cette lettre est consultable dans la rubrique « Actualités » du site, titre « processus qualité ».

35. Nous avons souhaité préserver l'anonymat de l'établissement étudié, ainsi que des personnes 
rencontrées. Nous avons choisi cet établissement car sans être pionnier dans le domaine des Tic, il a, dès les années 2000, développé en interne un espace de travail dit collaboratif, puis a opté pour une solution nationale d'ENT ainsi qu'une plate-forme d'apprentissage en ligne, sous licence open source.

36. De nombreux termes sont utilisés : « intranet», « espace de travail collaboratif», «espace collaboratif», «environnement numérique de travail ». Dans le cas de l'établissement étudié, un portail intranet permet d'accéder à des ressources (les textes de référence pour les marchés publics, les lois et règlements), à des actualités, au plan achat micro lié à l'opération «MiPE, micro portable étudiant » initié par le ministère de l'Éducation nationale en 2004 et à l'espace collaboratif. En interne, l'espace collaboratif est majoritairement désigné par le nom du produit ou par son sigle ou par le terme « intranet » qui ne permet cependant pas de distinguer le portail de l'espace collaboratif.

37. IATOS : Ingénieurs, Administratifs, Techniciens et Ouvriers de service.

38. Cf. appel à projets campus numérique volet 2 de 2003.

39. Site Tice de l'établissement étudié, texte disponible depuis février 2007 (site consulté en septembre 2008).

40. Voir Isabelle Bruno, À vos marques, prêts... cherchez !, la stratégie européenne de Lisbonne, vers un marché de la recherche, Éditions du croquant, savoir/agir, Paris, 2008.

41. Christine Barats, «Discours de l'excellence et effets des classements internationaux : l'exemple des universités parisiennes (changements de noms et logique de marque) », à paraître.

42. Michel Foucault, L'ordre du discours, Gallimard, 1971 : «le discours n'est pas simplement ce qui traduit les luttes ou les systèmes de domination, mais ce pour quoi, ce par quoi on lutte, le pouvoir dont on cherche à s'emparer. », p. 12.

43. Perspective pragmatique, telle que l'envisageait Austin, Quand dire c'est faire, Seuil (How to do things with words, 1962), 1970. 
$R \cdot E ́ \cdot S \cdot U \cdot M \cdot E ́$

Dans un contexte de mutation et de réformes du supérieur, l'étude des textes ministériels sur l'intégration des Tic indique une rhétorique de l'internationalisation éducative caractérisée par la notion de «compétitivité ». La compétitivité attendue du supérieur français réactualise l'injonction technologique comme "compétence culturelle et économique » indispensable. Elle souligne d'autre part la légitimité accordée aux discours et techniques issues des sciences dites de gestion et du management. La diffusion et la circulation de notions comme celles de « démarche qualité » et de « bonnes pratiques », au niveau national (autorité de tutelle), comme au niveau local (un établissement) attestent de la publicisation du dire managérial qui s'avère porté par les Tic, tout autant que ces notions contribuent à produire le dire managérial et à le banaliser.

In a context marked by mutations and reforms of the Higher Education system, the study of ministerial texts on the ICT's integration shows for the educative internationalization a rhetoric marked by the notion of «competitiveness». The expected competitiveness of French Higher Education brings back the technological injunction as an indispensable «cultural and economic competence». It underlines also the legitimacy attributed to discourses and technics taken from gestion and management sciences. The diffusion and circulation of notions as «quality demarche» and «good practices», at the national (tutelar autority) as well as local (an institution) levels, witness the publicisation of managerial speech which reveals to be supported by the ICT, while those notions contribute to produce the managerial speech and to trivialize it. 
